\title{
Research Agenda for Microbiome Based Research for Multidrug-resistant Organism Prevention in the Veterans Health Administration System
}

\author{
Ashley E. Kates, PhD; ${ }^{1,2}$ Jessica S. Tischendorf, MD; ${ }^{1,2}$ Marin Schweizer, $\mathrm{PhD} ;^{3,4,5}$ Loreen Herwaldt, MD; ${ }^{3,4}$ \\ Matthew Samore, MD; ${ }^{6,7}$ Kimberly C. Dukes, PhD; ${ }^{5,8}$ Dale N. Gerding, MD; ${ }^{9,10}$ Daniel J. Diekema, MD, MS; ${ }^{4,11,12}$ \\ Nasia Safdar, MD, $\mathrm{PhD}^{1,2}$
}

(See introductory commentary by Livorsi et al, pages 186-188.)

Nearly 100 trillion microbes occupying the skin, genitourinary tract, and pulmonary system constitute the human microbiome. ${ }^{1}$ This diverse microbiome consisting of bacteria, fungi, parasites and protozoa plays a significant role in health and disease. Impairment of the gut microbiome is implicated in a diverse array of conditions, both noninfectious and infectious, such as cardiovascular disease, ${ }^{2}$ Crohn's disease, ${ }^{3,4}$ and Clostridium difficile infection (CDI).,

Improvements in sequencing technology and decreases in sequencing costs have facilitated growth in microbiome research. Projects like the Human Microbiome Project ${ }^{7}$ and the European Metagenomics of the Human Intestinal Tract (MetaHIT $)^{8}$ have contributed enormously to our understanding of microbiome complexity and its role in health and disease. Despite this welcome progress, microbiome research is in its infancy, particularly in the context of how the microbiomes influences infection with multidrug resistant organisms (MDRO), including CDI in healthcare settings. Further research aimed at understanding and manipulating the structure and function of the microbiome in human physiology, disease pathogenesis, and infection prevention is necessary.

As the largest integrated healthcare system in the United States, serving 8.9 million veterans, the Veterans Health Administration (VHA) system is poised to lead innovative microbiome research in healthcare-associated infections and MDROs. Here, we discuss the proceedings of a multidisciplinary conference on microbiome research in this area across the VHA. This conference highlighted the role of the human microbiome in colonization with MDROs and infection prevention and developed recommendations to guide
VHA microbiome research in healthcare-associated infection (HAI) and MDROs (Table 1). The importance of a standardized approach to microbiome research, the role of the gut microbiome with special focus on biotherapeutics, and the role of microbiota at extraintestinal sites are the central focus of these recommendations. While the VHA is uniquely positioned to lead such microbiome research efforts, these proceedings have application beyond the VHA as well.

\section{RECOMMENDATIONS}

\section{A Framework for Microbiome Research}

A VHA-wide standardized research framework will ensure rigorous, reproducible work and foster collaborations across VHA sites. The need for a set of standards in microbiome research is not unique to the VHA. ${ }^{1,9}$ Several methodologies have been applied at all stages of microbiome analysis; however, a gold standard has not emerged. The International Human Microbiome Standards (IHMS) project ${ }^{10}$ was established to create a set of standard operating procedures (SOPs), which are crucial to enhancing reproducibility and comparability across microbiome research. Despite the importance of the proposed standards, many labs have not adopted these SOPs. ${ }^{9}$ As the VHA advances microbiome research, a standardized research framework must be developed. The recommended components of this framework are shown in Figure 1.

To ensure high-quality, reproducible microbiome research, a set of standardized methods should be implemented, such as the IHMS SOPs, which offer clinical definitions, sample collection best practices, and laboratory processes. Novel indices, such as a Microbiome Disruption Index being jointly designed

\footnotetext{
Affiliations: 1. William S. Middleton Memorial Veterans Affairs Hospital, Madison, Wisconsin; 2. Division of Infections Disease, Department of Medicine, University of Wisconsin School of Medicine and Public Health, Madison, Wisconsin; 3. Department of Epidemiology, College of Public Health, University of Iowa, Iowa City, Iowa; 4. Department of Internal Medicine, Carver College of Medicine, University of Iowa, Iowa City, Iowa; 5. Iowa City Veterans Affairs Health Care System, Iowa City, Iowa; 6. Division of Epidemiology, School of Medicine, University of Utah, Salt Lake City, Utah; 7. VA Salt Lake City Health Care System, Salt Lake City, Utah; 8. Institute for Clinical and Translational Research, University of Iowa, Iowa City, Iowa; 9. Edward Hines Jr Veterans Affairs Hospital, Hines, Illinois; 10. Loyola University Chicago Stritch School of Medicine, Maywood, Illinois; 11. Office of Clinical Quality, Safety and Performance Improvement, University of Iowa Hospitals and Clinics, Iowa City, Iowa; 12. Division of Medical Microbiology, Department of Pathology, University of Iowa Carver College of Medicine, Iowa City, Iowa.

Received November 13, 2017; accepted December 2, 2017

(c) 2018 by The Society for Healthcare Epidemiology of America. All rights reserved. 0899-823X/2018/3902-0011. DOI: 10.1017/ice.2017.311
} 
TA вLE 1. Proposed Veterans Health Administration Health Services Research and Development Agenda for Microbiome Research

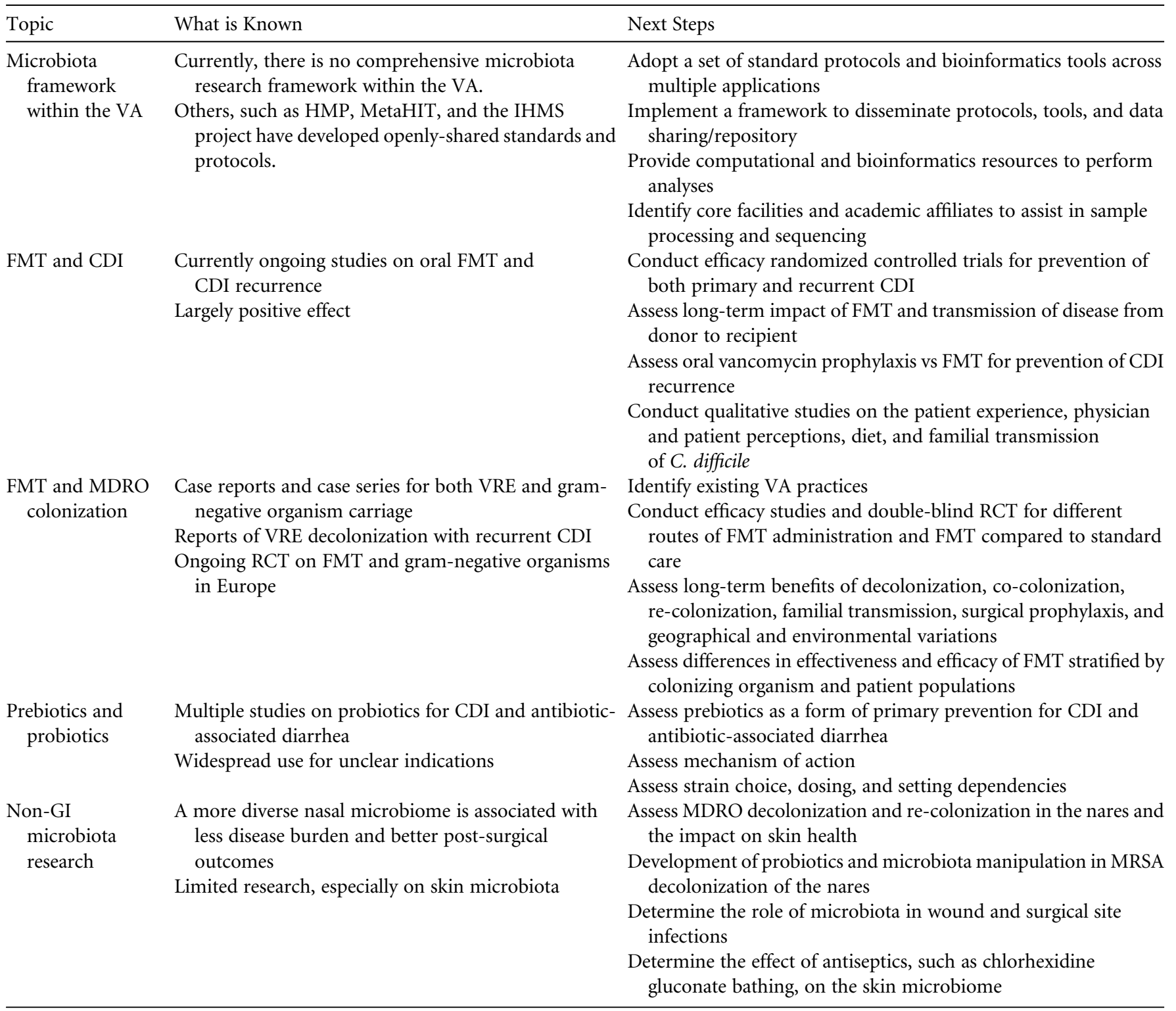

NOTE. VA, Veterans Affairs; HMP, Human Microbiome Project; IHMS, International Human Microbiome Standards; CDI, Clostridium difficile infection; FMT, fecal microbiota transplantation; MDRO, multidrug-resistant organism; VRE, vancomycin-resistant Enterococcus; RCT, randomized controlled trial; GI, gastrointestinal; MRSA, methicillin-resistant Staphylococcus aureus.

by uBiome and the Centers for Disease Control and Prevention (CDC), ${ }^{11}$ may offer promise in tracking the impact of interventions and biotherapeutics on the gut microbiome, can provide early identification of MDRO colonization or infection, and can serve as a method to standardize classification based on set definitions. While a greater understanding of microbiome compositions is required to create standardized classifications, some metrics do currently exist, such as the community states used to classify the vaginal microbiome. ${ }^{12}$

Presently, sample collection procedures vary widely and are dependent on the research question, the anatomic site, the expected biomass, and preferences of the research participant. The cost of the technique and the necessary sample size also influence the sampling procedure. While it is not possible to identify a set of sampling techniques appropriate for all studies, it would be beneficial to curate a set of guidelines and best practices.

Uniformity in laboratory processing methods will optimize the ability to compare results across studies. The method of genome extraction shapes the observed microbial composition due to varying cell lysis techniques. ${ }^{13}$ Standardizing methods of genome extraction will require access to core facilities or academic affiliates to carry out sequencing. For VA medical centers without these capabilities, collaboration with a core facility equipped to handle genome extraction is prudent and economical.

Resources for data analysis must be provided to microbiome researchers. Advanced computational and bioinformatics tools 


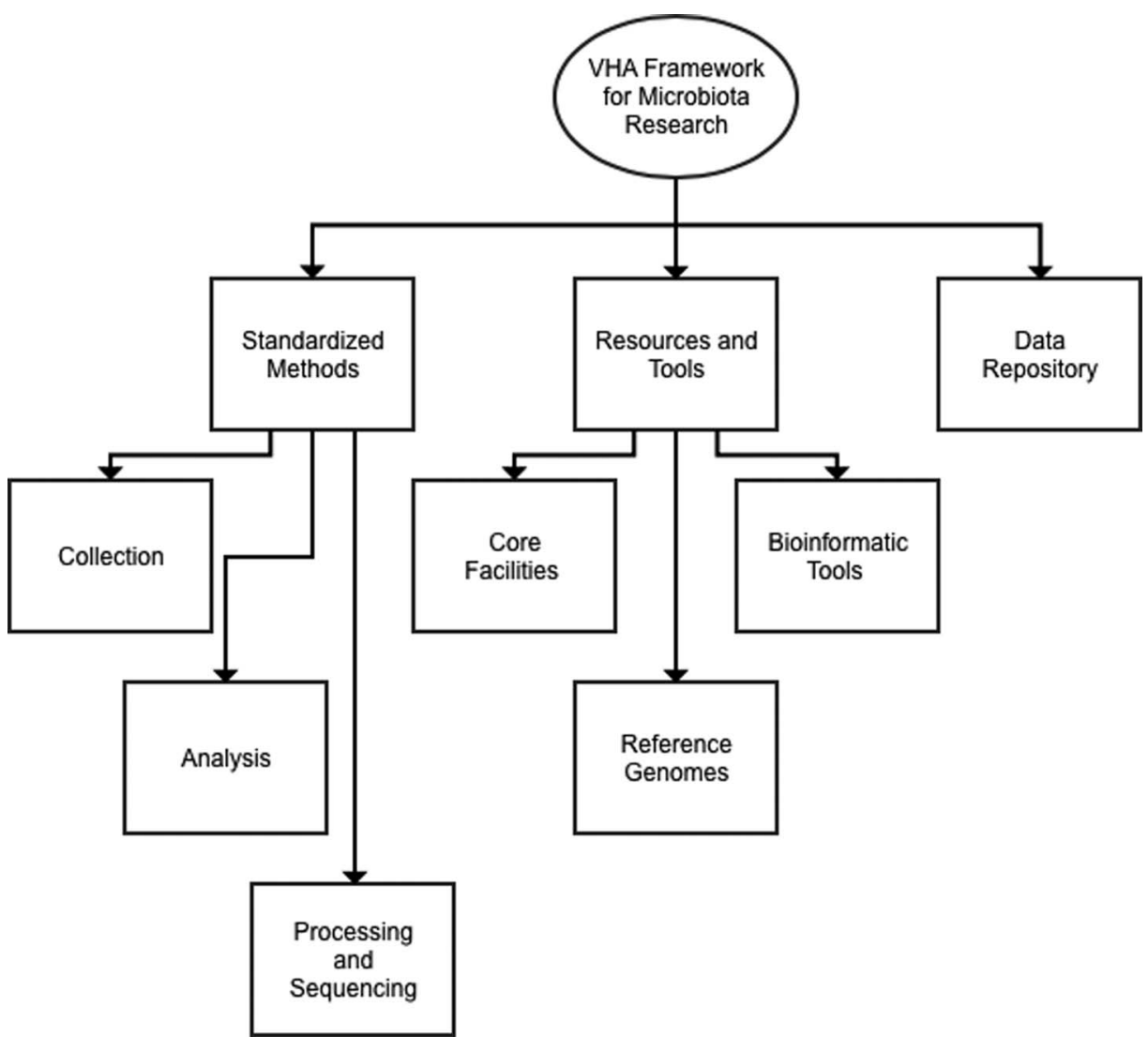

FIGURE 1. Key components of the proposed framework for microbiota research within the Veterans Health Administration (VHA).

are needed to analyze the volume of data produced through next-generation sequencing and shotgun metagenomics studies. Many tools currently exist for analyzing sequencing data, such as Quantitative Insights Into Microbial Ecology (QIIME) ${ }^{14}$ mothur, ${ }^{15}$ and $\mathrm{UPARSE}^{16}$ for targeted amplicon sequencing. However, gaps remain in our understanding of the relative advantages and disadvantages of these methods. After sequencing data are processed, statistical software $(R$ is one such example) must be available for data analysis. ${ }^{17}$ In addition to providing access to these programs, the VHA could create a central infrastructure of new and innovative analytic methods to be at the forefront of microbiome research and big data analysis in this context.

Data release and sharing into a repository is essential for microbiome research to ensure reproducibility and validation and allow leveraging of existing or planned studies. Sequencing and metadata should be uploaded to the repository frequently and following normal standards ${ }^{18}$ so it can be available to the scientific community. Repository data should be reasonably deidentified, consistent with institutional review board policies.

\section{Study Design Considerations}

Much of the published literature on the human microbiome and interventions to treat perturbations of the human microbiome are case studies or uncontrolled case series. ${ }^{19}$ The VHA can advance microbiome research by pursuing longitudinal studies, conducting randomized controlled trials (RCTs) of biotherapeutic interventions, investigating geographic differences in the microbiome, examining patient and provider acceptability of biotherapeutics through qualitative methods, and leveraging the Million Veterans Program as a considerable potential biobank for microbiome data.

Longitudinal studies can elucidate the natural history of changes in the microbiome across time, across the healthcare continuum, and in response to various exposures. Changes in the microbiome should be studied across transitions into and out of the hospital, intensive care units, long-term care facilities, community living centers, and pre- and postoperatively. ${ }^{20}$ By comparing groups of patients taking certain medications of interest (eg, antibiotics, metformin), changes to the microbiome related to these exposures can be identified. The influence of disease processes such as diabetes on the microbiome can also be studied in this way. ${ }^{21}$ The VHA can evaluate the influence of deployment on the microbiome by following the microbiome of military personnel who travel to and from other countries. These longitudinal studies must compare individuals exposed to the variable of interest to those who are not and take care to collect data on other variables that may affect the composition of the microbiome, such as diet, use 
of antibiotics, and occupation. These variables should be evaluated as risk factors for microbiome perturbations, and adjusted for in analyses.

Randomized controlled trials evaluating the effectiveness of biotherapeutic interventions to limit microbiome perturbation or restore the gut microbiome must be performed. These interventions include, but are not limited to, fecal microbiota transplantation (FMT), probiotics, and nontoxigenic C. difficile. Randomized controlled trials evaluating the use of these biotherapeutics in primary and recurrent CDI and other MDROs should be pursued. ${ }^{19}$ Because these biotherapeutics are organic, studies should account for potential transmission between study subjects, such as nontoxigenic spores transmitted from intervention and control patients.

Both longitudinal studies and RCTs need to be sufficiently powered to detect clinically relevant differences. A recent review by Hanson and Weinstock ${ }^{22}$ provides a discussion of methods that can be used for these power calculations.

The VHA should use its strong qualitative and implementation research infrastructure to assess patient and provider views regarding biotherapeutic interventions to alter the microbiome. Patients along the spectrum of health and disease should be surveyed or interviewed to determine attitudes and perceptions toward various types of biotherapeutics and delivery methods. ${ }^{19}$ These studies could reveal differences in risk perception and aversion to biotherapeutics among individuals based on prior disease or carrier status. Similarly, provider perceptions and how they approach conversations regarding these biotherapeutic interventions should be explored.

The VHA system is uniquely poised to perform many of the studies described above. Deployment to developing countries and the ability to connect TriCare data with the VHA Corporate Data Warehouse can provide much of the information needed to perform decades-long longitudinal studies with little added burden to the study subjects. Research to determine geographic variation of microbiomes will be necessary to define a "healthy microbiome" and whether interventions need to be geographically specific, which is important for veterans deployed internationally. The shared electronic medical record across inpatient, outpatient, and community living center care within the VHA provides the opportunity to follow patients during transitions of care. The VHA research funding mechanisms can also encourage investigators to add microbiome analyses to studies of antibiotics, decolonization interventions and new devices.

The Million Veteran Program (MVP) could provide a wealth of information to advance microbiome research. As of August 2016, the MVP had collected blood samples for genetic analysis and has performed baseline and lifestyle (eg, dietary habits, environmental exposures) surveys on more than 500,000 veterans. ${ }^{23,24}$ These data are linked with the electronic medical record to create a mega-biobank to improve understanding of how health is affected by genetics, behaviors, and environmental factors. ${ }^{23}$ The MVP could also collect stool samples from these Veterans and store them in the mega-biobank, allowing researchers to evaluate how health is affected by the microbiome and how behaviors and environmental factors may be associated with perturbations of the microbiome. By conducting longitudinal observational studies, RCTs and leveraging currently existing resources, the VHA can lead innovative research aimed to fully understand the gut microbiome so it can be leveraged for veteran and civilian health.

\section{INTERVENTIONS TARGETED TOWARD THE MICROBIOME}

\section{Fecal Microbiota Transplantation}

Gut dysbiosis is the hallmark of both initial and recurrent CDI. Conventional treatments include antibiotics with activity against $C$. difficile, ${ }^{25,26}$ but these antibiotics have activity against other gut bacteria, limiting the ability of the microbiota to fully recover following CDI and predisposing patients to recurrence. ${ }^{27}$ Although other factors may also explain why patients have recurrence (eg, low serum antibody response to C. difficile toxins ${ }^{28}$ use of medications such as proton pump inhibitors, ${ }^{29}$ and the specific strain of $C$. difficile causing infection $^{26,29}$ ), restoration of the gut microbiome through FMT has recently gained acceptance as a safe and effective treatment for CDI. However, the optimal protocol for FMT is unknown; numerous methods of stool preparation, infusion, and recipient and donor preparation have been published. ${ }^{30}$ Variation exists in diluent selected, site of instillation, method of recipient preparation, and donor screening. Fecal microbiota transplantation has been performed in both inpatient and outpatient settings, and self-administration of fecal enema at home has also demonstrated success. ${ }^{31}$ Although there are numerous variables to consider in protocol design, it is encouraging that FMT appears to be effective regardless of the specific protocol. ${ }^{32}$ Studies to evaluate the efficacy and the implementation of FMT for CDI are needed to define best practices.

Several key areas were identified as VHA priorities including efficacy RCTs for both primary and recurrent CDI, long-term assessment of the impact of FMT and transmission of disease from donor to recipient, assessment of oral vancomycin prophylaxis versus FMT for prevention of CDI recurrence, and qualitative studies on the patient experience and provider and patient perceptions.

Recurrent CDI. The clinical evidence for FMT is most robust for recurrent CDI, and 3 RCTs in this field have helped to clarify the efficacy of FMT. ${ }^{33-35}$ Each found rates of cure greater than $70 \%-90 \%$ following FMT; however, sample size, methodologic limitations, need for multiple FMT in several patients and limited duration of follow-up in these studies means there remains a need for high-quality prospective trials.

Primary and severe CDI. Few data are available on the use of FMT for primary, non-recurrent CDI aside from a few case reports. A mathematical model of CDI in an ICU assessed the role of FMT on primary $\mathrm{CDI}^{36}$ and predicted a decreased median incidence of recurrent CDI in patients treated with 
FMT for primary CDI. The assumptions of the model require validation and prospective testing to address the role of FMT in primary CDI. In a nonrandomized, open-label, before-andafter prospective study comparing conventional antibiotic treatment for CDI versus early FMT via nasogastric infusion, Lagier et $\mathrm{al}^{37}$ found that mortality in the FMT group was significantly less $(64.4 \%$ vs $18.8 \% ; P<.01)$. This shift in therapy occurred due to clinical need; their hospital in Marseille developed a ribotype 027 outbreak with a dramatic global mortality rate $(50.8 \%)$. In an epidemic setting with a high mortality rate, early FMT may be beneficial, but the risks and benefits of FMT in primary CDI in endemic situations have not been well described. Similarly, the evidence for use of FMT in severe CDI consists of published case reports, which suggest efficacy and potential for further study. ${ }^{38-41}$

Patient and provider perceptions of FMT. A commonly cited reason for a limited role of FMT is the aesthetics of treatment. However, few studies exist on patients and provider perceptions regarding FMT. In their 2014 review of FMT for CDI, the Evidence-based Synthesis Program (ESP), a unit of the VA Health Services Research and Development Service, identified no study designed to systematically assess acceptability of FMT among patients. The ESP highlighted these investigations as important next steps in advancing the FMT research agenda. ${ }^{42}$

Zipursky et $\mathrm{al}^{43}$ surveyed 192 outpatients (only 1 had a history of CDI) on their attitudes toward FMT using hypothetical case scenarios. The results were largely positive, with $81 \%$ agreeing to FMT for CDI. The need to handle stool and the nasogastric route of administration were the most unappealing aspects of FMT by participants. More respondents $(90 \% ; P=.002)$ agreed to FMT when offered as a pill.

When the same investigators surveyed physician attitudes toward FMT, ${ }^{44}$ they found 83 of 135 physicians $(65 \%)$ in their sample had not offered or referred a patient for FMT. Frequently cited reasons included institutional barriers, concern patients would find it unappealing, and uncertainty regarding indications. Only $8 \%$ of physicians believed patients would choose FMT if given the option. As the role of FMT expands, patient and provider perceptions and attitudes will likely evolve to better align.

FMT for MDROs. The hypothesized mechanism of FMT for CDI is that normal colonic microbiota outcompete and competitively exclude $C$. difficile. ${ }^{35}$ The same principles may apply to other enteric pathogens, such as vancomycin-resistant Enterococcus (VRE). Similar to C. difficile, VRE colonization is induced by microbial dysbiosis. Introduction of commensal microbiota may help eliminate VRE colonization through direct effects such as nutrient depletion or immune-mediated factors. In murine models, FMT is effective at decolonizing VRE. ${ }^{45,46}$ In case studies of patients co-colonized with C. difficile and VRE, FMT has resulted in the decolonization of both pathogens. ${ }^{47,48}$ However, rigorous evidence of efficacy is lacking, and determining the role of FMT for VRE decolonization requires randomized controlled trials.
Fecal microbiota transplantation may have the potential to reduce colonization with carbapenem-resistant Enterobacteriaceae (CRE). In several small case series, FMT successfully eradicated $\mathrm{CRE}^{49,50}$ In a recent single-center study of 20 patients receiving FMT for recurrent CDI, DNA microarray analysis identified decreased number and diversity of antibiotic resistance genes, which persisted up to a year following FMT. ${ }^{51}$ The availability of oral FMT makes it an attractive, relatively low-risk option for eradicating MDRO colonization and warrants further study.

\section{B I O T H E R A P E U T I C S}

The role of prebiotics, probiotics, and synbiotics (mixtures of pre- and probiotics) in the human gastrointestinal (GI) microbiome requires further examination. Prebiotics can be harnessed to enhance community structure and immune responses that confer protection against CDI. For example, fructooligosaccharide and lactulose prebiotics increase resistance to colonization with $C$. difficile via several mechanisms. The dairy protein glycomacropeptide (GMP) participates in activities related to GI physiology and metabolic disease and beneficially modulates GI microbiota in mice. ${ }^{52}$

When administered in adequate amounts, probiotics confer a health benefit. Several large systematic reviews have demonstrated a reduction in incidence of primary CDI when probiotics were used. ${ }^{51,53,54}$ Synbiotics, mixtures of probiotics and prebiotics, confer protection against CDI in mice, reducing viable $C$. difficile in cecal contents compared to controls. Current limitations to the probiotic literature are that many over-the-counter probiotics have no live organisms, do not have sufficient quantities of microbes, or have no mechanistic basis for their proposed action. These findings may explain the conflicting results from probiotic trials and the gap in our understanding of which probiotics are likely to confer benefit. A recent advance in this area is the use of nontoxigenic C. difficile spores. In a phase $2 \mathrm{RCT}$, patients who received non-toxigenic $C$. difficile spores following a resolved episode of CDI had a significant reduction in the risk of recurrence. ${ }^{55}$

The field of biotherapeutics is in its infancy and fundamental biomedical research is needed to understand the potential of these products before widespread implementation and dissemination. Trials of biotherapeutics offer an opportunity to examine GI microbiome changes as a consequence and better understand mechanisms of action.

\section{MICROBIOTA RESEARCH BEYOND THE GASTROINTESTINAL TRACT}

The gastrointestinal tract is the most studied human microbiome; however, many other body sites are of clinical relevance to HAI research, particularly the nasal and skin microbiomes.

Methicillin-resistant Staphylococcus aureus (MRSA) has been the focus of nasal microbiome research. MRSA colonization is associated with decreased nasal microbiome diversity and several bacterial species (Streptococcus mitis ${ }^{56}$ and 
Corynebacterium spp) influence MRSA colonization and virulence. ${ }^{57,58}$ Studying the effect of therapeutics applied in the nose and longitudinal studies of nasal microbiome changes during hospitalization, antibiotic use and after decolonization are necessary.

Studying the skin microbiome is challenging, but a better understanding of its role is necessary. Constant contact with the environment makes it difficult to distinguish between commensal and transient organisms, and obtaining adequate microbial DNA is challenging given the low biomass of the skin. While skin decolonization with daily or presurgical use of chlorhexidine to reduce infection is becoming more widespread, the impact of antiseptics on long-term skin health and microbial composition is unknown. In addition, the role of the skin microbiome in wound and surgical site infections and the impact of the hand microbiome of healthcare workers in MDRO transmission warrants further study.

\section{CONCLUSIONS}

The meeting of a multidisciplinary group of researchers focused on infection prevention and microbiota research identified several key recommendations for the future of microbiota research within the VHA. First, a unified framework including standardized methodologies, access to bioinformatics and computational resources, and provision of a data repository will allow high-quality, reproducible work and collaboration across multiple VHA sites. Incorporating microbiome data into data collection, particularly in infection prevention, will provide the ability to evaluate relationships of the microbiome to lifestyle and clinical factors. Longitudinal studies will elucidate changes in the human microbiome over time and across transitions of care. High-quality RCTs examining the efficacy of various approaches to FMT and qualitative studies exploring patient and provider preferences will clarify the role of FMT in restoration of health and treatment of disease. Additional biotherapeutics should be examined, such as prebiotics, probiotics and synbiotics. Finally, the role of extraintestinal microbiomes, particularly the nose and skin, in health and disease should be studied further.

Given the large role the VHA plays in the health of veterans and civilians and its strong research infrastructure, the VHA is well positioned to lead the next wave of discovery in human microbiome research.

\section{ACKNOWLEDGMENTS}

Financial support: This work was supported in part by funding from the VA Health Services Research and Development (HSR\&D) Service Center of Innovation (COIN) conference supplement ("Setting the Clinical Research Agenda for MDROs in VA," grant no. CIN 13-412) and by the VA Quality Enhancement Research Initiative CARRIAGE Program (grant no. IP1 HX001993-01A1). The views expressed in this article are those of the authors and do not necessarily reflect the position or policy of the Department of Veterans Affairs or the US government. DNG holds patents for treatment and prevention of CDI, is a member of Advisory Boards for Merck, Summit, Actelion, Rebiotix and DaVolterra and is a consultant for Sanofi Pasteur, MGB Pharma and Pfizer.
Address correspondence to Ashley Kates, $\mathrm{PhD}, 5^{\text {th }}$ Floor, UW Medical Foundation Centennial Bldg, 1685 Highland Ave, Madison, WI 53705 (akates@medicine.wisc.edu).

\section{REFERENCES}

1. Ravel J, Blaser MJ, Braun J, et al. Human microbiome science: vision for the future, Bethesda, MD, July 24 to 26, 2013. Microbiome 2014;2:16.

2. Tang WHW, Wang Z, Levison BS, et al. Intestinal microbial metabolism of phosphatidylcholine and cardiovascular risk. N Engl J Med 2013;368:1575-1584.

3. Pascal V, Pozuelo M, Borruel N, et al. A microbial signature for Crohn's disease. Gut 2017;66:813-822.

4. Pedamallu CS, Bhatt AS, Bullman S, et al. Metagenomic characterization of microbial communities in situ within the deeper layers of the ileum in Crohn's disease. Cell Molec Gastroenterol Hepatol 2016;2:563-566.e565.

5. Schubert AM, Rogers MAM, Ring C, et al. Microbiome data distinguish patients with Clostridium difficile infection and nonC. difficile-associated diarrhea from healthy controls. mBio 2014;5:e01021-01014.

6. Ferreyra JA, Wu KJ, Hryckowian AJ, Bouley DM, Weimer BC, Sonnenburg JL. Gut microbiota-produced succinate promotes C. difficile infection after antibiotic treatment or motility disturbance. Cell Host Microbe 2014;16:770-777.

7. Human Microbiome Project: overview. National Institutes of Health website. http://commonfund.nih.gov/hmp/overview. Published 2014. Accessed January 4, 2018.

8. Qin J, Li R, Raes J, et al. A human gut microbial gene catalogue established by metagenomic sequencing. Nature 2010;464:59-65.

9. Raising standards in microbiome research. Nature Microbiol 2016;1:16112.

10. International Human Microbiome Standards website. http:// www.microbiome-standards.org/. Published 2015. Accessed February 27, 2017.

11. The microbiome and innovations to slow antibiotic resistance. Centers for Disease Control and Prevention website. https:// www.cdc.gov/drugresistance/solutions-initiative/microbiomeinnovations.html. Published 2017. Accessed January 4, 2018.

12. Gajer P, Brotman RM, Bai G, et al. Temporal dynamics of the human vaginal microbiota. Science Translat Med 2012;4:132ra152-132ra152.

13. Kuczynski J, Lauber CL, Walters WA, et al. Experimental and analytical tools for studying the human microbiome. Nat Rev Genet 2012;13:47-58.

14. Caporaso JG, Kuczynski J, Stombaugh J, et al. QIIME allows analysis of high-throughput community sequencing data. Nat Methods 2010;7:335-336.

15. Schloss PD, Westcott SL, Ryabin T, et al. Introducing mothur: open-source, platform-independent, community-supported software for describing and comparing microbial communities. Applied Environ Microbiol 2009;75:7537-7541.

16. Edgar RC. UPARSE: highly accurate OTU sequences from microbial amplicon reads. Nat Meth 2013;10:996-998.

17. R: A language and environment for statistical computing. 2010. R Development Core Team website. http://www.r-project.org/. Published 2010. Accessed January 4, 2018.

18. The Wellcome Trust. Sharing Data from Large-scale Biological Research Projects: A System of Tripartite Responsibility. 2003; Fort Lauderdale: FL. 
19. Kociolek LK, Gerding DN. Breakthroughs in the treatment and prevention of Clostridium difficile infection. Nature Rev Gastroenterol Hepatol 2016;13:150-160.

20. Halpin AL, McDonald LC. Editorial commentary: the dawning of microbiome remediation for addressing antibiotic resistance. Clin infect Dis 2016;62:1487-1488.

21. Forslund K, Hildebrand F, Nielsen T, et al. Disentangling type 2 diabetes and metformin treatment signatures in the human gut microbiota. Nature 2015;528:262-266.

22. Hanson BM, Weinstock GM. The importance of the microbiome in epidemiologic research. Ann Epidemiol 2016;26:301-305.

23. Gaziano JM, Concato J, Brophy M, et al. Million Veteran Program: a mega-biobank to study genetic influences on health and disease. J Clin Epidemiol 2016;70:214-223.

24. US Department of Veterans Affairs Office of Research and Development. Million Veteran Program: for researchers and research partners. Department of Veterans Affairs website. https://www.research.va.gov/MVP/researchers.cfm. Published 2017. Accessed January 4, 2017.

25. Cohen SH, Gerding DN, Johnson S, et al. Clinical practice guidelines for Clostridium difficile infection in adults: 2010 update by the society for healthcare epidemiology of America (SHEA) and the infectious diseases society of America (IDSA). Infect Control Hosp Epidemiol 2010;31:431-455.

26. Crook DW, Walker AS, Kean Y, et al. Fidaxomicin versus vancomycin for Clostridium difficile infection: meta-analysis of pivotal randomized controlled trials. Clin Infect Dis 2012;55: S93-S103.

27. Chang JY, Antonopoulos DA, Kalra A, et al. Decreased diversity of the fecal microbiome in recurrent Clostridium difficileassociated diarrhea. J Infect Dis 2008;197:435-438.

28. Kyne L, Warny M, Qamar A, Kelly CP. Association between antibody response to toxin $\mathrm{A}$ and protection against recurrent Clostridium difficile diarrhoea. Lancet 2001;357:189-193.

29. Abou Chakra CN, Pepin J, Sirard S, Valiquette L. Risk factors for recurrence, complications and mortality in Clostridium difficile infection: a systematic review. PLoS ONE 2014;9:e98400.

30. Chapman BC, Moore HB, Overbey DM, et al. Fecal microbiota transplant in patients with Clostridium difficile infection: a systematic review. J Trauma Acute Care Surg 2016;81:756-764.

31. Silverman MS, Davis I, Pillai DR. Success of self-administered home fecal transplantation for chronic Clostridium difficile infection. Clin Gastroenterol Hepatol 2010;8:471-473.

32. Bakken JS, Borody T, Brandt LJ, et al. Treating Clostridium difficile infection with fecal microbiota transplantation. Clin Gastroenterol Hepatol 2011;9:1044-1049.

33. Youngster I, Russell GH, Pindar C, Ziv-Baran T, Sauk J, Hohmann EL. Oral, capsulized, frozen fecal microbiota transplantation for relapsing Clostridium difficile infection. JAMA 2014;312:1772-1778.

34. Cammarota G, Masucci L, Ianiro G, et al. Randomised clinical trial: faecal microbiota transplantation by colonoscopy vs. vancomycin for the treatment of recurrent Clostridium difficile infection. Aliment Pharmacol Therapeut 2015;41: 835-843.

35. van Nood E, Vrieze A, Nieuwdorp M, et al. Duodenal infusion of donor feces for recurrent Clostridium difficile. $N$ Engl J Med 2013;368:407-415.

36. Lofgren ET, Moehring RW, Anderson DJ, Weber DJ, Fefferman NH. A mathematical model to evaluate the routine use of fecal microbiota transplantation to prevent incident and recurrent Clostridium difficile infection. Infect Control Hosp Epidemiol 2013; 35:18-27.

37. Lagier JC, Delord M, Million M, et al. Dramatic reduction in Clostridium difficile ribotype 027 -associated mortality with early fecal transplantation by the nasogastric route: a preliminary report. Eur Soc Clin Microbiol 2015;34:1597-1601.

38. Neemann K, Eichele DD, Smith PW, Bociek R, Akhtari M, Freifeld A. Fecal microbiota transplantation for fulminant Clostridium difficile infection in an allogeneic stem cell transplant patient. Transplant. Infect Dis 2012;14:E161-E165.

39. Trubiano JA, Gardiner B, Kwong JC, Ward P, Testro AG, Charles PGP. Faecal microbiota transplantation for severe Clostridium difficile infection in the intensive care unit. Eur J Gastroenterol Hepatol 2013;25:255-257.

40. Gallegos-Orozco J, Paskvan-Gawryletz C, Gurudu S, Orenstein R. Successful colonoscopic fecal transplant for severe acute Clostridium difficile pseudomembranous colitis. Rev Gastroenterol Mex 2011;77:40-42.

41. You DM, Franzos MA, Holman RP. Successful treatment of fulminant Clostridium difficile infection with fecal bacteriotherapy. Ann Intern Med 2008;148:632-633.

42. Drekonja D, Reich J, Gezahegn S, et al. VA Evidencebased Synthesis Program reports. Fecal Microbiota Transplantation for Clostridium Difficile Infection: A Systematic Review of the Evidence. Washington, DC: Department of Veterans Affairs; 2014.

43. Zipursky JS, Sidorsky TI, Freedman CA, Sidorsky MN, Kirkland KB. Patient attitudes toward the use of fecal microbiota transplantation in the treatment of recurrent Clostridium difficile infection. Clin Infect Dis 2012;55:1652-1658.

44. Zipursky JS, Sidorsky TI, Freedman CA, Sidorsky MN, Kirkland KB. Physician attitudes toward the use of fecal microbiota transplantation for the treatment of recurrent Clostridium difficile infection. Can J Gastroenterol Hepatol 2014;28:319-324.

45. Caballero S, Carter R, Ke X, et al. Distinct but spatially overlapping intestinal niches for vancomycin-resistant enterococcus faecium and carbapenem-resistant Klebsiella pneumoniae. PLoS Pathogens 2015;11:e1005132.

46. Ubeda C, Bucci V, Caballero S, et al. Intestinal microbiota containing Barnesiella species cures vancomycin-resistant Enterococcus faecium colonization. Infect Immun 2013;81:965-973.

47. Halpin AL, de Man TJ, Kraft CS, et al. Intestinal microbiome disruption in patients in a long-term acute care hospital: a case for development of microbiome disruption indices to improve infection prevention. Am J Infect Control 2016;44:830-836.

48. Stripling J, Kumar R, Baddley JW, et al. Loss of vancomycinresistant enterococcus fecal dominance in an organ transplant patient with Clostridium difficile colitis after fecal microbiota transplant. Open Forum Infect Dis 2015;2:ofv078.

49. Davido B, Batista R, Michelon $\mathrm{H}$, et al. Is faecal microbiota transplantation an option to eradicate highly drug-resistant enteric bacteria carriage? J Hosp Infect 2017;95:433-437.

50. Lagier JC, Million M, Fournier PE, Brouqui P, Raoult D. Faecal microbiota transplantation for stool decolonization of OXA-48 carbapenemase-producing Klebsiella pneumoniae. J Hosp Infect 2015;90:173-174.

51. Millan B, Park H, Hotte N, et al. Fecal microbial transplants reduce antibiotic-resistant genes in patients with recurrent Clostridium difficile infection. Clin Infect Dis 2016;62:1479-1486. 
52. Sawin EA, De Wolfe TJ, Aktas B, et al. Glycomacropeptide is a prebiotic that reduces Desulfovibrio bacteria, increases cecal short-chain fatty acids, and is anti-inflammatory in mice. Am J Physio. Gastrointestin Liver Physiol 2015;309:G590-G601.

53. Johnston BC, Ma SY, Goldenberg JZ, et al. Probiotics for the prevention of clostridium difficile-associated diarrhea: a systematic review and meta-analysis. Ann Intern Med 2012;157:878-888.

54. Shen NT, Maw A, Tmanova LL, et al. Timely use of probiotics in hospitalized adults prevents Clostridium difficile infection: a systematic review with meta-regression analysis. Gastroenterol 2017;152:1889-1900.e1889.

55. Gerding DN, Meyer T, Lee C, et al. Administration of spores of nontoxigenic Clostridium difficile strain M3 for prevention of recurrent $C$. difficile infection: a randomized clinical trial. JAMA 2015;313:1719-1727.

56. Bessesen MT, Kotter CV, Wagner BD, et al. MRSA colonization and the nasal microbiome in adults at high risk of colonization and infection. J Infect 2015;71:649-657.

57. Ramsey MM, Freire MO, Gabrilska RA, Rumbaugh KP, Lemon KP. Staphylococcus aureus shifts toward commensalism in response to Corynebacterium species. Front Microbiol 2016; $7: 1230$.

58. Uehara Y, Nakama H, Agematsu K, et al. Bacterial interference among nasal inhabitants: eradication of Staphylococcus aureus from nasal cavities by artificial implantation of Corynebacterium sp. J Hosp Infect 2000;44:127-133. 